LA-UR-81-2969

$$
\text { MASTSTSP }
$$

TITLE

AUTOMATED SUPERNOVA SFARCH

AUTHOAISI

Stirling A. Colgate, T-6 suemitted To For publication in the Proceedings of the NATO-sponsored

"Advanre Study Instltute" on: Supernovac, Cambrldge, Engliand, June 29-July 10, 1981 


\title{
AUTOMATED SUPERNOVA SEARCH
}

\author{
Stirling A. Colgate \\ New Hexico Institute of Mining and Technology \\ Socorro, New Mexico 87801 \\ and \\ Los Alamos National Laboratory, University of Californio \\ Los Alamos, New Mexico 87545
}

\section{INTRODUETION}

A progran lior the developent of ari outomated supernova asarch based upon complete remote computer control of a telescope ant vidirsa digital inaging systee has been under developnent at New Hedico Institute of Hining and Technology, Socorro, New Mexico, since 1968. This very long tise has seen the multiple developent of many systeas as well as sone fallures. The priary problea has been the complexity of the software system. Although, in the early days several different digital image tubes were developed, ang which vere Orthicons and luocona, before the present aystes of an intensified Vidicon silicon target tube was put in place. ${ }^{2}$ The ooftware problea is. aure subtle problem than the single coaponents of hardware. 3 However, before going into boftunce io depth let us ronsider the ncientilic objectives.

One vould like to detect oupernovae at an early stage of their light curve in sufficient numbers to escablish some of the following criteria:

1. One would like to underntand if the eacly jight curve is derived from ahock wave in an extended envelope or by an interpol energy cource; for exanple, the rudioactive decay of $\mathrm{Ni}^{50}$ etc. 
2. One would like to understand if the luminosity at peak is consiztent with an internal energy source that may le radioactivity or by a hydrodynamic shock wave or early pulsar emission, and compare this to theories of the origin of supernovae, whether thermonuclear energy release or dynamic collapse to a neutron star state.

3. One would like to obtain sufficient statistics of the pea and width of all types of supernovae to understand the consistency of the initial state and the consistency of the surrounding environment of the supernova. For instance, type I supernova occurring in E-galaxies with negligible gas and dust may exhibit sufficiently repetitive light curves to serve as a secondary standard in the measurement of the size and curvature, $H_{0}, q_{0}$ of the universe. 15

4. One would like t\& oblain sufficient statistics of supernovae concerning their location within galaxies as well as the galaxy type, and ultimately extend that study to large enough $Z$ to determine possible evolutionary factor in the occurrence of supernova as measure of stellar evolution in galaxies as a function of time.

These questions are best served by a system that images galaxies sequentially and attempts to find a difference in the galaxy image past and in the present, indicative of a point source of light increasing in luminosity with time constant of days to weeks. This requires (1) atoring previous image and comparing it to a present one, (2) maintaining a catalogue of galaxies as well as part images, (3) a catalogue system that keeps track of false alarms and contains bufficient logic to deal with these in a hierarchy of exclusion tests, and (4) a system which can continue for long periods of time unattended because of the intense boredow of monitoring system that is performing a highly repetitive, although complex, function. It was therefore the objective from the beginning to develop atand-alone system of a telescope in a dark, good seeing site controlled through a microwave link from a computer on a campus where students could take turns in ultimately and hopefully the relatively routine function of computer operation.

This cystem amost reached operational status but was termiunted in 1974 for several reasons.

1. No furtber external funding was avallable.

2. The computer, IBM 360 , Model 44, was completing its natural life after 10 years at the institution and the meintenance requirements were beconing so excessive that negligible night time or off hours use was feasible.

3. Probably most inportant, the software biructure had become so complicated that debugging and modifying it became impractical because of the imense difficulty of tracing an unoperstive interaction throughout the whole satem, roughly 100,000 FORTRAN itatement in size. 
The question naturally arose whether the entire system should be dismantled. On the other hand, the hardware on the mountain, although constructed to a significant extent of surplus components, was still significantly beyond the state of the art. This included: lescope mount driven by digital stepping motors in a feedback mode that could slew the telescope a radian in several seconds of time with an accuracy of better than $10 \mathrm{~s}$ of arc. It used a special light-weight design with a self-supported central mirror and allowed the change of focal ratio from $F-30$ to F-12 to take place in less than $5 \mathrm{~s}$. It contained a fully automatió cross dispersed eschell spectograph and an intensified Vidicon digital image tube system that operated without attention remotely for many months at a time. In addition, a microwave link had been developed with a dropout rate of less than 1 in $10^{\circ}$ bits which was adequate for the purpose in queston. Finally, there was the relatively complicated decoding and query logic in place for all the many functions needed to operate such a system provided the appropriate 16-bit words were transmitted to the mountain. This seemed such a significant development in itself that the consideration of a computer with its software to direct this system secmed to be a feasible addition that could take place at a relatively modest level of investment over an extended period of time.

A possibility to do this arose with the funding to purchase - Prime 300 minicomputer for the joint use with the atmospheric sciences with the specific capabilities of multiuser and virtual memory operating system. The raason for this is the recognition that the software effort will be many times larger than the hardware effort. It therefore stands to reason that if you have one task 10 times greater than the others it is wise to have more than one person work on the problem simultaneously. This is why the operating system must be a multiuger viriual wi....y ine shared system. but also mutitasking where a separate task (like user) is assigned to each of several telescope functions; e.g., telescope slewing, the digital cameras (CCD or SIT), star field pattern recognition, spectra, guifing, frave "taking", reduction and calibration, calalogue access and asintenance of stars, zalaxies, quasars, and nebulae. A multitasking operating astem allows -ll of these fusctions to be operating concurrently provided a real time interrupt of the operatiisg system can take place. Most multiuser opersting systems asaign a sequential tibe slice to each user. A teacocope must be able to ccmand attention with a priority above nther tasks.

In oddition there must he:

1. User obwervation program testing, development and schedul ing.

2. User data formating and presentation. 
3. User inicesvention.

4. Interlocks, telescope safety, and fault diagnosis.

A multiuser environment allows any task to talk to another task by passing parameters much like the function "mail" where any user passes messages to other users.

Many may ask why not use a separate microcomputer for each task? One then has the problem of networking all these computers - which can be done but it is presently much more difficult again because the software effort is much greater than the hardware effort. It is much more efficient to time share one CPU that "knows" where everytting (everyone) is located and where the operating system is a development used for thousands of installations.

We have been modifying a Prime 300 (a 16-bit minicomputer with $256 \mathrm{~K}$ core and 80 Mbyte disc) multiuser time sharing system to handle priority interrupts so that we can perform multitasking in a real time environment.

Currently the Prime 300 operating system has been modified to perform priority interrupt real time transfer of data to and frow the mountain via microwave link. All programs are imbedded in mult-user environment. In addition, message passing between tasks has been implemented so that parameiers are easily available to all programs. Many otber system changes have been implemented to accomplish this and roughly $3 / 4$ of the operating routines have been rewritten and are operational.

A telephone mudern interface with call-back using the state Watts telephone system allows users to log in with a remote terminal.

\section{ACKNOWLEDGERENTS}

Bill Thompson ol Lawrence Livermore National Laboratory and Bryan Edwards of Prol nk, Bouider did the initial design of the Prime system. It has been seveloped and implemented by Eric Pearce and Kevin Meinr at New Mexico Tech. It is supported by New Mexico Institute of Mining and Technology, Los Alamos National Laboratory, and private support.

1. Moore, E. Merillat, P. Colgate, S., and Corlson, R., 1975: Software for a Digitally Controlled Telescope. Proceedings of the MiT Conference on Teleacope Autonation, pp. 91-107. ed. M. K. Huguenin and T. B. McCord.

2. Colgate, S. A., Moore, E. P., and Colburn, J. 1975, Applied Optics, 14, 1429. 
3. Colgate, S. A., Moore, E. P, and Ca.lson, R., 1975, Publications of the Astronomical Society of the Pacific, 87, 565-575.

4. Wagoner, R. V., 1977, Ap. J., 214, L5.

5. Colgate, S. A 1979 , Ap. J., $2 \overline{32}, 404-408$.

6. Colgate, S. A., and Thompson, III, W. C. 1980. Ground Based Automated Telescope. Presented at optical and infrared Program, Tucson, Arizona, January 7-12, 1980 - TELESCOPES FOR THE 1980's.

$\mathrm{SAC} /$ rep : 36B 